\title{
Multilinguales
}

$10 \mid 2018$

Langues et médias dans les milieux plurilingues

\section{Le code switching en Algérie : les langues autour du français radiophonique}

Code-switching in Algeria: the languages surrounding French of radio

broadcasts

\section{Nabil Sadi}

\section{(2) OpenEdition}

\section{Journals}

Édition électronique

URL : http://journals.openedition.org/multilinguales/3618

DOI : $10.4000 /$ multilinguales.3618

ISSN : 2335-1853

Éditeur

Université Abderrahmane Mira - Bejaia

Référence électronique

Nabil Sadi, «Le code switching en Algérie : les langues autour du français radiophonique »,

Multilinguales [En ligne], 10 | 2018, mis en ligne le 31 décembre 2018, consulté le 03 juin 2020. URL http://journals.openedition.org/multilinguales/3618; DOI : https://doi.org/10.4000/multilinguales. 3618

Ce document a été généré automatiquement le 3 juin 2020

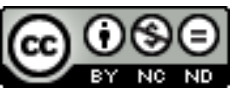

Multilinguales est mise à disposition selon les termes de la Licence Creative Commons Attribution -

Pas d'Utilisation Commerciale - Pas de Modification 4.0 International 


\title{
Le code switching en Algérie : les langues autour du français radiophonique
}

\author{
Code-switching in Algeria: the languages surrounding French of radio \\ broadcasts
}

Nabil Sadi

1 «Le paysage sociolinguistique en Algérie se caractérise par l'existence de plusieurs langues (et variétés de langue) régies par des règles historiques, sociopolitiques et culturelles bien déterminées » (Sadi, 2012: 60). Ces langues en contact engendrent des pratiques langagières spécifiques sur les plans formel et structurel, régies par divers facteurs d'ordre discursif et situationnel.

Dans ce sens, Queffélec et al. (2002) soulignent que

La société algérienne étant plurilingue, ce contact des langues se traduit par des comportements langagiers très particuliers mais tout à fait naturels pour ce type de société. Les idiomes s'interpénètrent au gré des relations sociales, des stratégies discursives des locuteurs et de leurs compétences linguistiques et surtout en fonction du caractère formel et/ou informel de la situation de communication. Cette interpénétration s'accompagne d'une instabilité dans l'utilisation des codes linguistiques en présence, instabilité marquée essentiellement par la coexistence de différents idiomes dans une même séquence: les locuteurs passent très souvent d'une langue aux autres, mettant en contact dans la linéarité du discours les vernaculaires usités- l'arabe algérien et/ou les variétés du berbère et les langues académiques- l'arabe standard/l'arabe classique et le français. (112)

$2 \mathrm{Au}$ cours des différentes interactions, les locuteurs algériens utilisent leurs compétences langagières pour divers besoins de communication. Barillot (2002) en identifie le code switching. Pour désigner ces énoncés où apparaît ce phénomène de changement ou d'alternance de langues, ou de variétés linguistiques dans un discours ou une conversation, les linguistes ont proposé toute une série de termes qui traduisent l'abondance et la variété des approches. Si certains chercheurs reprennent la terminologie anglo-saxonne et utilisent code-switching (terme inventé par Haugen dès 
1956), code-mixing ou code-changing, d'autres recourent aux concepts de formation française comme alternance codique (Gumperz, 1989b, traduit par Simonin, 2001), alternance des codes (Hamers et Blanc), alternance des langues (Gardner-Chloros, 1983 ; 1985 ; 1991), métissage linguistique (Sesep N’sial, 1979), (Queffélec et al., 1997).

Ce phénomène a fait l'objet de nombreuses études se réclamant d'écoles, d'orientations et d'approches différentes. Il a aussi été qualifié de discours mélangé (Nissaboury, 1999), de rencontre d'habitudes linguistiques (Charnet, 1999), de heurt de deux langues (Lafont, 1997), perçu comme une pratique langagière déviante, marginale et transitoire (Gumperz 1989), entendu comme une manifestation d'un bilinguisme idéal (Weinreich, 1953), ou encore en tant qu'un signe de décadence linguistique (Hoffmann, 1991), d'inculture ou de mutilation linguistique (Mabrour. A, 2007: 12-20). Par conséquent, les définitions de l'alternance codique abondent au point de s'opposer parfois.

Nous reprenons dans ce travail la dénomination code switching en proposant d'étudier la structure lexicale qui découle de cette pratique et de voir quelles sont les langues qui alternent le plus avec le français dans un contexte radiophonique interactionnel, l'ordre d'apparition de cette alternance et les différentes formes qu'elle peut prendre.

4 L'analyse que nous proposons portera sur trois émissions radiophoniques qui constituent notre corpus, à savoir Le rendez-vous de l'économie, Conseils et vous et Micro ondes. Ces émissions d'Alger-chaine 3 se caractérisent par la diversité des thèmes ainsi que par les différents locuteurs, des professionnels et des non-professionnels de la radio, des personnes du métier, des protagonistes et antagonistes.

\section{La structure des langues en contact : autour du français}

5 Nous avons choisi d'examiner une seule émission à la fois, vu que les réalisations sont diverses, que les langues alternées sont différentes et varient d'une émission à une autre et d'un locuteur à un autre, afin d'en dégager les points communs ou les divergences. Comme le souligne Charaudeau (2001: 7) : "Analyser c'est toujours essayer de voir ce qui dans la différence apparente est ressemblant et ce qui dans la ressemblance apparente est différent ».

\section{Analyse de l'émission $1:$ Le rendez-vous de l'économie}

6 La première émission se caractérise par une alternance de deux langues. Ce phénomène touche quatre langues différentes qui n'alternent jamais à la fois. Nous avons noté l'alternance du français et du kabyle, l'alternance du français et de l'arabe, l'alternance $\mathrm{du}$ français et de l'anglais.

7 L'ordre d'apparition des langues alternées diffère, et la forme de cette alternance varie selon les langues.

\section{L'alternance français-kabyle (F+K)}

Nous avons recensé un seul passage où le français alterne avec le kabyle. Il s'agit de l'animateur assistant qui salue son collègue en français et poursuit en affirmant sa présence en kabyle. 
Comme nous l'avons déjà mentionné, l'animateur lance le débat, organise les tours de parole, tandis que son assistant pose les questions pertinentes. En atteste l'extrait suivant :

$\mathrm{I}_{\mathrm{A}}$ : bonsoir Hamid aqlagh dayi (on est là)

\section{L'alternance français-arabe $(\mathrm{F}+\mathrm{Ar})$}

En ce qui concerne l'alternance du français et de l'arabe, trois formes apparaissent ${ }^{1}$. Nous avons noté des passages où les locuteurs prennent la parole en français, introduisent l'arabe et poursuivent leurs phrases en français $(\mathbf{F}+\mathbf{A r}+\mathbf{F})$. Il s'agit de quelques invités et un auditeur :

$\mathrm{I}_{1}$ : nous avons donc des bureaux des wilayat (divisions administratives de l'Algérie) à l'ensemble euh sur l'ensemble du territoire national

$\mathrm{I}_{2}$ : dans des pays qui se respectent// les les walis (préfets) ou les maires vous vous mettent un tapis rouge

$\mathrm{I}_{3}$ : une destination touristique pas euh à à la mode/ qui qui va émerger pendant quelques années/ oumbaad (ensuite) qui va tomber dans la banalité

$\mathrm{A}_{\mathrm{U} 2}$ : tout à l'heure vous avez fait la comparaison bine (entre) euh la route transsaharienne et l'autoroute

Dans les deux premiers passages, les deux invités introduisent, dans leur discours en français, deux emprunts arabes de la même famille. Par contre, les deux derniers passages se caractérisent par l'utilisation de connecteurs en arabe dans un discours en français $(\mathbf{F}+\mathbf{C A r}+\mathbf{F})$. Ces deux connecteurs relient les deux propositions en français.

11 Quatre autres passages se caractérisent par l'alternance du français et de l'arabe, où les quelques invités et un auditeur commencent à parler en français et terminent en arabe $(\mathrm{F}+\mathrm{Ar})$ :

$\mathrm{I}_{2}$ : son mektoub (destin)

$\mathrm{I}_{1}$ : c'est quoi exactement encouragement yaâni (c'est-à-dire)

$\mathrm{I}_{4}$ : franchement la première euh remarque qui vient à l'esprit des gens/ c'est que ah $\uparrow$ khlas tabachir (fini la craie)

$\mathrm{A}_{\mathrm{U} 2}$ : alors ou vous me laissez dire ce que j'ai à dire entièrement oulla ma neqbelch (si non je refuse)

Le même type d'alternance apparaît dans trois passages, mais cette fois-ci, l'arabe apparait en première position suivi du français $(\mathbf{A r}+\mathbf{F})$ :

$\mathrm{I}_{\mathrm{A}}$ : aandna (nous avons) les mêmes problèmes que les Etats-Unis?

$\mathrm{I}_{3}$ : ourahoume (où sont-ils) les investissements touristiques dont ils parlaient ?

$\mathrm{A}_{\mathrm{U} 2}$ : el moqarana bine (la comparaison entre) les routes en bitume et les routes en béton

Les deux premières interrogatives (directe et indirecte) se limitent à deux lexies en arabe, tandis que dans la troisième phrase, le segment en arabe est plus long.

\section{L'alternance français-anglais $(F+A n)$}

Pour ce qui est de l'alternance du français et de l'anglais, une seule forme se distingue avec une forte apparition du français en première position, suivi de l'anglais en deuxième position (F+An). En attestent les exemples suivants :

$\mathrm{I}_{2}$ : Martin Luther King/ avait dit un certain temps/ I have a dream (je fais ce rêve) 
$\mathrm{I}_{2}$ : ceux qui ont fait les études euh ont prévu un remblaiement directement sur un ouvrage qui est réalisé sur un spike (pointe)

$\mathrm{I}_{2}$ : euh parce qu'il faut commencer quand même d'un euh firm strip (commerce)

$\mathrm{I}_{4}$ : et dire que nous sommes plus un peu dans le euh le be to be (présentiel)

$\mathrm{I}_{4}$ : il y a des logiciels/ qui font mieux circuler l'information en reporting (report)

À chaque fois que les invités décident d'alterner le français et l'anglais, ils commencent toujours leurs énoncés en français. Aucun passage d'alternance n'a été enregistré chez l'animateur principal (A).

\section{Analyse de l'émission 2 : Conseils et vous}

$14 \mathrm{Au}$ cours de la deuxième émission, l'alternance codique touche deux langues uniquement, à savoir le français et l'arabe. Trois formes sont à souligner également quant à l'ordre d'apparition des langues alternées.

\section{L'alternance français-arabe $(\mathrm{F}+\mathrm{Ar})$}

La langue de base étant le français, l'arabe apparaît généralement en deuxième position $(\mathbf{F}+\mathbf{A r})$. Nous avons recensé 13 passages où les auditeurs semblent s'approprier cette forme :

$\mathrm{A}_{\mathrm{U} 3}$ : ça y est $\uparrow$ les unités khlasou (il y'en a plus)

A : oh lala $\uparrow$ alors les unités khlasou (il y'en a plus)/

$\mathrm{A}_{\mathrm{U} 1}$ : les lignes sont saturées oullah ghaleb (dommage)

$A_{U 1}$ : quand il est chez moi c'est euh c'est hadja (quelque chose)

$\mathrm{A}_{\mathrm{U} 2}$ : pour lui lazem neqaad feddar ybellaâ alia ma nekhredj ma nahder maa heta ouahed (il faut que je reste à la maison, la porte fermée, que je ne sorte pas et que je ne parle à personne)

$\mathrm{A}_{\mathrm{U} 2}$ : il y a des gens yaayrouni zaâma (c'est-à-dire ils se moquent de moi)

$\mathrm{A}_{\mathrm{U} 3}$ : ils m'ont accepté mais kima n'goulou f'el maslaha $\uparrow$ (comme on dit c'est par intérêt)

$\mathrm{A}_{\mathrm{U} 3}$ : quand quelqu'un te voit pleurer/ il se euh yedjbed rouhou menek (il s'éloigne de toi)

$\mathrm{A}_{\mathrm{U} 3}$ :j'ai j'ai euh kima n'goulou gadra $\uparrow$ (comme on dit capable)

$\mathrm{A}_{\mathrm{U} 3}$ : voilà $\uparrow /$ c'était rien ou le tout/ c'est là que j'ai commencé blaaqel blaaqel (petit à petit)

$A_{U 3}$ : ça va pas être facile/ mais maâliche (c'est pas grave)

$\mathrm{A}_{\mathrm{U} 3}$ : je passe le grand bonjour à aami (mon oncle) Noureddine

$\mathrm{A}_{\mathrm{U} 5}:$ il faut mettre le hidjab (voile)

Mis à part l'animateur qui alterne (à une seule reprise) le français et l'arabe dans le deuxième passage, en reprenant simplement les propos de son interlocuteur $\left(\mathrm{A}_{\mathrm{U} 3}\right.$ dans le premier passage), cette pratique de poursuivre en arabe un discours en français caractérise uniquement les auditeurs.

Les auditeurs de la deuxième émission, jouent le rôle principal dans le déroulement des interactions, et déterminent par conséquence la structure des alternances codiques relevées au cours des échanges.

16 La deuxième forme de l'alternance du français et de l'arabe, à savoir commencer en arabe et poursuivre la phrase en français $(\mathbf{A r}+\mathbf{F})$, se retrouve également chez les 
auditeurs, même si ce n'est pas aussi répandu que la première forme. En voici les extraits :

$\mathrm{A}_{\mathrm{U} 3}$ : ki ychoufek tdahki (quand il te voit sourire) et tout euh tout le monde s'approche de toi $\uparrow$ / et se comporte normal

$A_{U 1}$ : laâbad berk elli (les gens seulement qui) euh qui appellent pour nous dire pour nous encourager// pour ces petits conseils qu'on/ qu'on communique

$\mathrm{A}_{\mathrm{U} 1}$ : yaâni (c'est-à-dire) du fond du cœur/ euh elle va nous manquer cette émission

$\mathrm{A}_{\mathrm{U} 3}$ : nqoulha (je lui dirai) c'est pas la fin du monde

Dans ces quatre passages, les auditeurs construisent des énoncés bilingues en passant de l'arabe au français, que ce soit d'une proposition à une autre ou d'une lexie à une proposition.

Dans le deuxième numéro, les auditeurs usent de connecteurs arabes pour lier des propositions en français $(\mathbf{F}+\mathbf{C A r}+\mathbf{F})$. Ces connecteurs se résument à des lexies, considérées comme des petits mots :

$\mathrm{A}_{\mathrm{U} 1}$ : et là je voudrais euh je voudrais tant que ma participation apporte yaâni (ça veut dire) un euh un bénéfice pour euh toute la famille algérienne

$\mathrm{A}_{\mathrm{U} 3}$ : il a pas euh il n'a pas chghel (comme) quelqu'un qui euh qui peut l'aider

$\mathrm{A}_{\mathrm{U} 3}$ : je vous ai dit qu'on est chghel (comme) financièrement intellectuellement euh on est chghel (comme) euh bien sûr il y a ma mère qui nous a appris cette idée qu'on était supérieurs quoi

$\mathrm{A}_{\mathrm{U} 3}$ : il y a mes oncles que euh que leurs femmes chghel (comme) ne travaillent pas euh chghel (comme) n'ont pas réussi dans leurs études

Comme nous pouvons le remarquer, l'auditeur $3\left(\mathrm{~A}_{\mathrm{U} 3}\right)$ introduit le même connecteur arabe « chghel » au milieu de propositions en français. Cette lexie semble apparaitre à des moments d'hésitation et quand l'auditeur cherche ses mots. En général, ce connecteur est précédé ou suivi de « euh » d'hésitation.

C'est la même fonction que joue "yaâni " dans le premier passage, où le premier auditeur poursuit son idée en français en passant par ce connecteur arabe.

\section{Analyse de l'émission 3 : Micro ondes}

Le thème de la troisième émission la rend plus spécifique que les précédentes. Consacrée aux tubes (tous genres confondus) qui ont marqué les années soixante-dix et quatre-vingts, Micro ondes se caractérise par la coexistence de plusieurs langues, à savoir le français, l'arabe, l'anglais et l'italien. Ces quatre langues entretiennent une relation des plus complexes et s'interpénètrent au fur et à mesure des échanges.

Cette imbrication se traduit par plusieurs formes, à savoir l'alternance du français et de l'arabe, du français et de l'anglais, du français et de l'italien, voire l'alternance de trois langues dans le même discours: le français, l'arabe et l'anglais/ le français, l'arabe et l'italien. Quant à l'ordre d'apparition des langues alternées, plusieurs schèmes sont à noter.

19 S'agissant de l'alternance du français et de l'arabe, nous avons relevé quatre structures différentes que nous exposerons une par une: 


\section{L'alternance français-arabe $(\mathrm{F}+\mathrm{Ar})$}

\section{français à l'arabe (F+Ar), à côté de deux auditeurs de deux numéros différents.}

Les prises de parole de l'animateur sont à base du français et commencent généralement par cette même langue quand il s'agit d'alterner. Nous avons recensé quatre passages où cette structure apparaît chez l'animateur et deux chez deux auditeurs :

A : il y a le policier qui lui dit ouathaiq esyara (papiers du véhicule)

$\mathrm{A}$ : je suis là samedi dimanche et lundi/ ala el qanat athalitha faqat ala el haoua oua ala el mobachir (sur la chaîne trois uniquement à l'antenne et en direct)

$\mathrm{A}$ : un véritable tabac en mille neuf cent// rebaa ou tmanyine (quatre-vingt-quatre)

A : bonne boussa (bise)

$\mathrm{A}_{\mathrm{U} 10}$ : peut-être pas la Sabrina que je connais mais balak (peut-être)

$\mathrm{A}_{\mathrm{U} 10}$ : et aussi Karim ouldi (mon fils)

L'alternance chez les auditeurs se résume à une lexie introduite à la fin de l'énoncé.

Pour ce qui est de la structure arabe-français $(\mathbf{A r}+\mathbf{F})$, le même auditeur du cinquième numéro alterne cette fois-ci deux propositions ; la première est en arabe tandis que la deuxième est en français. À côté de l'animateur qui inverse aussi l'ordre de l'alternance dans le même numéro :

$\mathrm{A}_{\mathrm{U} 10}$ : arouah neddik (viens je t'emmènerai) à la pêcherie

$\mathrm{A}:$ ah $\uparrow$ tdirouri (vous me ferez) du bon poisson

Une autre caractéristique propre aussi à l'animateur est à souligner, à savoir qu'il lie deux propositions, qu'elles soient exprimées en arabe ou en français, en introduisant des lexies puisées dans l'autre système. Observons ces trois extraits :

A : tahmima aala (une douche pour) euh comme ça aala tsourti (pour moi)

A : un peu de hnana (tendresse) dans ce monde de bruts

A : c'est hammam (douche) climatisé

En effet, dans le premier extrait, l'animateur introduit "comme ça ", un connecteur que nous pouvons également considérer comme un petit mot ou une forme d'hésitation, au milieu de deux propositions en arabe (Ar+CF+ Ar).

Par ailleurs, les deux lexies dans les deux autres extraits remplissent une autre fonction. Elles lient deux propositions en français $(\mathbf{F}+\mathbf{A r}+\mathbf{F})$ et complètent le sens de la phrase sans pour autant nuire à sa structure.

\section{L'alternance français-anglais $(F+A n)$}

La deuxième langue qui alterne avec le français dans cette troisième émission est l'anglais. La majorité des chansons qui défilent dans le Top Ten est en anglais, par conséquent plusieurs termes et/ou segments sont introduits au cours des énoncés en français, dont la plus grande partie apparaît sous la forme d'alternance français-anglais (F+An).

Cette pratique courante réside apparemment chez l'animateur, avec néanmoins un seul passage de la part d'un auditeur. Dix-huit (18) extraits font ressortir ce mélange du français et de l'anglais :

$\mathrm{A}_{\mathrm{U} 6}$ : il est too much (autant)

A: ah non $\uparrow$ vacances tel week (semaine) 
$\mathrm{A}$ : et puis après bien sûr/ le rappel en vitesse grand $\mathrm{v}$ de notre Top Ten (top dix)

A : on quitte les eighties (années quatre-vingts)

A : on quitte les nineties $\uparrow$ (années quatre-vingt-dix)

A : il passait en rotation/ sur toutes les stations radios du monde et sur tous les plateaux of television (de télévision)

A : on appelle ça les l'Italian touch (sens italien)

A : une figure emblématique bien sûr de euh song of the seventies (chanson des années soixante-dix)

$\mathrm{A}:$ il nous reste encore dix minutes à passer en votre company (compagnie)

A : un numéro un $\uparrow / /$ qui se pointe dans les charts (hit-parades)

$\mathrm{A}:$ la bande à mark relieve (signe de relève)

$\mathrm{A}$ : un mélange de Pop Rock new age (nouvelle ère)

$\mathrm{A}:$ bravo $\uparrow /$ tu viens de gagner one night in Algiers (une nuit à Alger)

A : six voix seulement pour le génie de l'électronique/ mister (monsieur) Moby

A : si je vous disais Talk Talk (parler parler)

Mis à part le premier passage où l'auditeur termine en anglais ce qu'il a commencé en français, les autres passages renvoient à ce que l'animateur fait tout au long de l'émission, à savoir animer, distraire et créer de l'ambiance tout en restant dans la politique et l'objectif de l'émission. Il poursuit le plus souvent en anglais en faisant allusion à des lieux, à des personnages et à des faits.

Un seul passage témoigne de l'ordre inversé des deux langues en question où l'animateur prend la parole en anglais en faisant référence au café et poursuit pour terminer en français :

A : coffee (café) de euh de dix-sept heures quarante-six

L'animateur introduit également à deux reprises des lexies en anglais au milieu d'énoncés en français. Cela se passe spontanément grâce à des segments en anglais ayant une valeur de qualificatifs :

A : un autre bad boy (mauvais garçon) des années soixante-dix quatre-vingts

A : un certain homme head (chef) / a fait un tabac/ avec ses quarante-cinq tours

\section{L'alternance français-italien/espagnol $(\mathrm{F}+\mathrm{l} / \mathrm{E})$}

La dernière structure relevant de l'alternance de deux langues concerne le français et l'italien/espagnol. La seule forme à noter est celle où l'animateur termine ses phrases en français avec des lexies en italien (F+I/E). Cela est aussi en étroite corrélation avec la devise de l'émission et de son atmosphère gaie. Deux noms en italien sont les plus utilisés par l'animateur et à maintes reprises. Nous avons choisi de représenter un seul exemple pour chaque nom:

A : ah oui/ alors vacances

à la playa (plage)

$\mathrm{A}:$ il s'agit de notre dixième et dernière nostalgia $\uparrow$ (nostalgie)

A : ils se reforment après vingtaine d'années de separazione (séparation)

En effet, mis à part "separazionne " qui n'est mentionné qu'une seule fois par l'animateur, "playa " et "nostalgia " sont repris plusieurs fois et dans chaque numéro.

Pour ce qui est de l'alternance de trois langues, deux extraits témoignent du mélange de l'arabe avec le français, l'anglais et l'italien. Nous avons relevé un passage où 
l'alternance touche l'arabe, le français et l'anglais, et un autre qui touche aussi les deux premières et l'italien (dans l'ordre cité), et c'est toujours chez le même locuteur.

\section{L'alternance arabe-français-anglais $(\mathrm{Ar}+\mathrm{F}+\mathrm{An})$}

\section{A : mafratche (ce n'est par réglé) pour Top Ten (top dix)}

Dans ce passage, l'animateur use d'un connecteur en français pour lier deux segments de deux langues différentes $(\mathrm{Ar}+\mathrm{CF}+\mathrm{An})$.

\section{L'alternance arabe-français-italien $(\mathrm{Ar}+\mathrm{F}+\mathrm{I})$}

\section{A : el qanat athalitha (la chaîne trois) pour la nostalgia (nostalgie)}

C'est le même procédé qu'on retrouve chez le même locuteur dans cet extrait. L'animateur introduit un connecteur en français pour lier cette fois-ci une proposition en arabe et une lexie en italien $(\mathbf{A r}+\mathbf{C F}+\mathbf{I})$.

Suite à cette analyse regroupant la structure lexicale de l'alternance codique conversationnelle dans trois émissions radiophoniques d'Alger-Chaîne III, nous pouvons remarquer que ce phénomène fait ressortir plusieurs formes et plusieurs structures, caractérisant ainsi chaque émission avec les diversités qu'elles recouvrent, quant au thème abordé dans chacune, l'objectif et les participants.

Ces différents paramètres qui constituent la situation de communication déterminent les langues en présence dans les interactions, conditionnent la forme sous laquelle apparaît le phénomène de l'alternance codique et régissent sa structure.

Nous proposons de synthétiser, sous forme d'un tableau (Zaboot, 1990), les modes de structuration relevés dans chaque émission :

Tableau 1 : Les modes de structuration dans Le rendez-vous de l'économie

\begin{tabular}{|l|l|l|l|l|}
\hline $\begin{array}{l}\text { Enoncés } \\
\text { Langues }\end{array}$ & Passage 1 & Connecteur (c) & Passage 2 & Passage 3 \\
\hline Arabe (Ar) & $\mathrm{Ar}$ & & $\mathrm{F}$ & \\
\hline Français (F) & $\mathrm{F}$ & $\mathrm{Ar}$ & K/Ar/An & F \\
\hline Kabyle (K) & & & K & \\
\hline Anglais (An) & & & An & \\
\hline
\end{tabular}

Tableau 2 : Les modes de structuration dans Conseils et vous

\begin{tabular}{|l|l|l|l|l|}
\hline $\begin{array}{l}\text { Enoncés } \\
\text { Langues }\end{array}$ & Passage 1 & Connecteur (c) & Passage 2 & Passage 3 \\
\hline Arabe (Ar) & A & & F & \\
\hline Français (F) & F & Ar & Ar & F \\
\hline
\end{tabular}


Tableau 3 : Les modes de structuration dans Micro ondes

\begin{tabular}{|l|l|l|l|l|}
\hline $\begin{array}{l}\text { Enoncés } \\
\text { Langues }\end{array}$ & Passage 1 & Connecteur (c) & Passage 2 & Passage 3 \\
\hline Arabe (Ar) & Ar & F & F/Ar/An/I & \\
\hline Français (F) & F & & Ar/An/I & F \\
\hline Anglais (An) & & & An & \\
\hline Italien (I) & & & I & \\
\hline
\end{tabular}

Nous remarquons que les différents énoncés qui résultent du phénomène de l'alternance codique conversationnelle présents dans notre corpus sont caractérisés par leur instabilité structurelle. Néanmoins, nous sommes arrivés à faire ressortir des similitudes et en même temps des divergences entre les trois émissions. Les locuteurs pratiquent ce phénomène dans chacune d'elles, mais chacun d'eux le fait d'une manière différente, d'une émission à l'autre et d'un thème à l'autre.

En somme, l'une des pratiques communes, c'est que les deux langues qui alternent le plus dans les trois émissions sont le français et l'arabe. Le français, étant la langue de base d'une chaîne radiophonique censée être francophone, cohabite le plus souvent avec l'arabe dialectal qui est l'idiome pratiqué par la plus grande partie de la population et

qui est avant tout la langue de la gestion de la quotidienneté (...) et par excellence l'outil de l'expression des différents rapports interpersonnels (...). Il couvre les domaines de la vie familiale, de l'affectivité, des sentiments, des états d'âme et de la religiosité du sujet parlant. (Queffélec et al., 2002 : 121-122)

Dans les deux premières émissions, le français est en première position, suivi le plus souvent de l'arabe. Ce dernier sert parfois aussi de connecteur entre deux passages en français. Mais ce qui différencie les deux émissions, c'est le nombre et le type de langues qui alternent, dans la mesure où nous observons une faible alternance du kabyle et de l'anglais avec le français dans la première, tandis qu'il y a, dans la seconde, alternance unique du français et de l'arabe.

Plusieurs langues cohabitent également dans la troisième émission. Cette fois-ci, ce sont le français et l'arabe qui apparaissent le plus souvent en première position, et chacune de ces deux langues sert à lier l'autre lorsqu'il s'agit de trois segments.

Même si ce sont généralement les langues qui alternent le plus, comme dans les précédentes émissions, l'anglais et l'italien marquent une faible alternance avec le français et l'arabe. Lorsqu'elles sont introduites dans une phrase, c'est en dernière position.

Nous constatons également que les locuteurs ont recours dans leur pratique à des connecteurs. Dans la plupart des énoncés, les connecteurs sont empruntés à un système linguistique différent de celui dans lequel sont rendus les énoncés qu'ils relient (en général, l'arabe et le français); si les deux passages sont en français, le connecteur sera rendu en arabe. 
Inversement, si les deux passages sont exprimés en arabe, ils seront reliés par un connecteur en français. Mais dans de rares cas, le connecteur qui est puisé dans une troisième langue, sert à lier deux langues différentes. Il s'agit de la dernière émission, où le français lie l'arabe avec l'anglais et l'italien.

Ce qui accroche vraiment, concernant les divergences entre les trois émissions, c'est l'impact de la composante "participants » sur ce phénomène. Nous avons constaté qu'au cours des deux premières émissions, ce sont les non professionnels de la radio qui semblent exceller dans cette pratique, à savoir les invités et les auditeurs. Les animateurs produisent un discours en français (le caractère formel de la chaîne), influencent les autres participants quant à la langue qu'ils utilisent d'un tour de parole à un autre, mais ils restent performants quant à l'utilisation d'une seule langue dans une même prise de parole.

La composante "thème » rentre en interaction avec ce postulat, dans la mesure où nous retrouvons l'animateur de la troisième émission qui utilise plusieurs langues dans son discours et qui ne cesse d'alterner deux, voire trois langues, pour répondre à des besoins stratégiques et communicationnels relatifs au thème de la musique et à l'objectif de l'émission. Il fait exception à la règle et rejoint les non professionnels qui usent de toutes leurs compétences langagières afin de mieux faire passer le message et de s'adapter au paysage des émissions.

\section{BIBLIOGRAPHIE}

BARILLOT, N., « Codeswitching arabe marocain/français : remarques générales et aspect prosodique ", in Comment les langues se mélangent. Codeswitching en francophonie, C. Canut \& D. Caubet (Eds.), L'Harmattan, pp. 119-134, 2002.

CHARAUDEAU, P., Aspects du discours radiophonique, Didier Erudition, Paris, 2001.

CHARNET, Ch., « "Ana je pose la question” ou quelques façons de parler de locuteurs marocains : étude de manifestation du contact linguistique ", in Manzano F. (Dir.), Les Langues du Maghreb et du sud méditerranéen, Rennes, P.U.R., 1999.

GARDNER-CHLOROS, P.H., « Code switching : approaches principales et perspectives », in La linguistique, vol. 19, fasc. 2, pp. 21-53, 1983.

GARDNER-CHLOROS, P.H., Choix et alternance des langues à Strasbourg, Thèse de Doctorat, Université Louis Pasteur Strasbourg, 1985.

GARDNER-CHLOROS, P.H., Language selection and switching in Strasbourg, Oxford, Clarendon Press, 1991.

GUMPERZ, J., Sociolinguistique interactionnelle- Une approche interprétative, Editions L'Harmattan, Paris, $1989 \mathrm{~b}$.

HAMERS, J.-F. \& BLANC, M.H.A., Bilinguisme et bilingualité, Editions Mardaga, Bruxelles, 1983.

HAUGEN, E., Bilingualism in the Americas, University of Alabama $\operatorname{Pr}(\mathrm{Tx})$, première édition, 1956. 
HOFFMANN, Ch., An Introduction to Bilinguisme, London, Longman, 1991.

LAFONT, R., Quarante ans de sociolinguistique à la périphérie, Paris, L'Harmattan, 1997.

MABROUR, A., Précis du plurilinguisme et du pluriculturalisme. Lettre Langue française, diversité culturelle et linguistique, 2007.

NISSABOURY, A., « Arabophones et francophones du Maroc : un bilinguisme dynamique », in Revue québécoise de linguistique, $\mathrm{n}^{\circ} 1,1999$.

QUEFFELEC, A., DALOBA, J. \& WENEZOUI-DECHAMPS, M., « Français et sango en contact : de l'interférence au discours mixte », Le français en Centrafrique- Lexique et Société, EDICEF, 1997, « Disponible sur http://www.bibliotheque.refer.org/livre11/accueil.htm » (Consulté le

11/01/2019).

QUEFFELEC, A., DERRADJI, Y., DEBOV, V., SMAALI-DEKDOUK, D. \& CHERRAD-BENCHEFRA, Y., Le français en Algérie, lexique et dynamique des langues, De Boeck \& Larcier s. a., Éditions Duculot, 1ère édition, 2002.

SADI, N., «L'usage du français à la chaîne 3 (aspects syntactico-sémantiques ", Thèse de doctorat en sciences, option sciences du langage, université de Bejaia, 2012.

SESEP N'SIAL, B.S., «Quelques hypothèses pour une définition du métissage linguistique », Langage et société, 9, pp. 32-46, 1979.

SIMONIN, J., GUMPERZ, J.-J., Sociolinguistique interactionnelle. Une approche interprétative, L'Harmattan, Paris, 2001.

WEINREICH, U., Languages in contact, La Haye, Mouton, 1953.

ZABOOT, T., Un code-switching algérien : le parler de Tizi-ouzou, Thèse de Doctorat de linguistique, Université René Descartes Paris-V, 1990.

\section{NOTES}

1. Nous avons choisi de ne pas reprendre les passages identiques, où s'alternent les mêmes lexies et les mêmes propositions. Seules les formes nous intéressent.

\section{RÉSUMÉS}

La présente étude analyse le discours radiophonique pratiqué en Algérie en dégageant les caractéristiques formelles du phénomène du code switching qui y intervient. Nous nous intéressons en particulier à la dimension structurelle de ce phénomène qui relève du domaine du plurilinguisme. Le contexte radiophonique (Alger-Chaîne 3) y joue un rôle capital, dans la mesure où le français est au contact de plusieurs langues véhiculaires d'Algérie.

The present study analyses the radio discourse practiced in Algeria by emphasising the formal characteristics of the phenomenon of code switching that interfere. We are particularly interested in the structural dimension of this practice, which is part of the field of 
multilingualism. The radio context of Alger-Chaîne 3 plays a key role, as French is in contact with several languages of communication.

\section{INDEX}

Keywords : code-switching, language contact, radio discourse, structural dimension, formal dimension

Mots-clés : code switching, contact de langues, discours radiophonique, dimension structurelle, structure formelle

\section{AUTEUR}

NABIL SADI

Laboratoire LESMS, Université de Bejaia, Algérie 